\title{
Determinantes do envelhecimento bem-sucedido
}

\author{
Ana Cristina Canêdo, ${ }^{1}$ Roberto A. Lourenço ${ }^{2 *}$
}

\section{Resumo}

Introdução: Nas últimas décadas, a expectativa de vida tem aumentado de forma significativa em muitos países. Porém, para que esta transformação demográfica seja sustentável em termos econômicos, sociais e de saúde pública, é preciso que a longevidade ocorra paralelamente à preservação da saúde e funcionalidade. Assim, a promoção do envelhecimento bem-sucedido tem sido tema de interesse crescente tanto no cenário das políticas públicas quanto nos estudos sobre envelhecimento humano. Objetivos: Discutir os fatores determinantes e preditores do envelhecimento bem-sucedido. Métodos: Revisão narrativa com busca de artigos nos bancos PubMed e SciElo utilizando os descritores: "envelhecimento bem-sucedido", "envelhecimento saudável", "determinantes do envelhecimento" e "preditores de envelhecimento". Resultados e Discussão: Do ponto de vista fisiológico, o envelhecimento varia amplamente entre os indivíduos, sendo que algumas pessoas apresentam extensas alterações fisiológicas e outras apresentam poucas ou nenhuma. Parte desta variabilidade pode ser explicada pela heterogeneidade genética (25\%) e estima-se que $75 \%$ seja determinado por fatores extrínsecos, como fatores ambientais, comportamentais, psicológicos inflamatórios, endócrinos e sociais. As variáveis com maior evidência de associação com envelhecimento bem-sucedido são idade, doenças articulares, audição, atividades de vida diária e tabagismo. Conclusões: A implementação de estratégias efetivas para a promoção do envelhecimento bem-sucedido exige que se desenvolva maior conhecimento sobre os seus fatores determinantes: tanto aqueles relacionados à taxa de declínio, quanto os fatores protetores para a manutenção da vitalidade e resiliência ao estresse.

Descritores: Envelhecimento bem-sucedido; Envelhecimento saudável; Determinantes; Preditores.

\section{Abstract}

\section{Determinantes do envelhecimento bem-sucedido}

Introduction: In the last decades, life expectancy has increased significantly in many countries. However, for this demographic transformation to be economically, socially and publicly sustainable, longevity must take place in parallel with the preservation of health and functionality. Thus, promotion of successful aging has been a topic of growing interest both in the context of public policy and in studies on human aging. Objectives: To discuss the predictor factors on successful aging. Methods: A narrative review including article search on PubMed and SciElo databases, using the keywords: "successful aging", "healthy aging" and "aging predictors". Results and Discussion: From a physiologic perspective, it is possible to affirm that aging varies widely among individuals, which
1. Serviço de Geriatria. Hospital Universitário Pedro Ernesto. Universidade do Estado do Rio de Janeiro. Rio de Janeiro, RJ, Brasil.

2. Departamento de Medicina Interna. Hospital Universitário Pedro Ernesto. Universidade do Estado do Rio de Janeiro. Rio de Janeiro, RJ, Brasil.

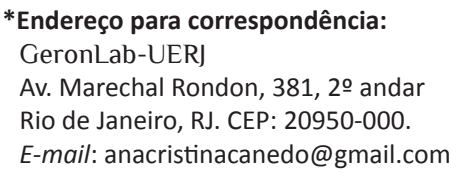

means that while some people have extensive psychological changes with age, others have few or none. Some of this variability can be explained by genetic heterogeneity (25\%), and there is an estimated $75 \%$ of this change that results from extrinsic factors, such as environmental, behavioral, psychological, inflammatory, hormonal, and social. The variables with most evidence of association with successful aging are age, articulation disorders, hearing, activities of daily living and smoking. Conclusions: In order to implement effective successful aging strategies, it is mandatory to develop a better knowledge about the predictor factors: both those related to the declining rate and those related to the protecting factors responsible for keeping the vitality and resilience to stress.

Keywords: Successful aging; Healthy aging; Predictors.

\section{Resumen}

\section{Determinantes del envejecimiento exitoso}

En las últimas décadas, la expectativa de vida ha aumentado de forma significativa en muchos países. Sin embargo, para que esta transformación demográfica sea sostenible en términos económicos, sociales y de salud pública, es necesario que la longevidad ocurra paralelamente con la preservación de la salud y la funcionalidad. Por lo tanto, la promoción del envejecimiento exitoso, ha sido tema de interés creciente tanto en el escenario de las políticas públicas como en los estudios sobre envejecimiento humano. Objetivos: Discutir sobre los factores predictores del envejecimiento exitoso. Métodos: Una revisión narrativa incluye la búsqueda de artículos en las bases de datos PubMed y Scielo, usando las palabras clave: "envejecimiento exitoso", "envejecimiento saludable" y "predictores del envejecimiento". Resultados y Discusión: Desde una perspectiva fisiológica, se puede afirmar que el envejecimiento 
varía ampliamente entre los individuos, lo que significa que mientras algunas personas presentan extensos cambios fisiológicos con la edad, otros presentan pocas o ninguna. Parte de esta variabilidad puede ser explicada por la heterogeneidad genética (25\%) y se estima que el $75 \%$ de este cambio es el resultado de factores extrínsecos como ambientales de comportamiento, psicológicos, inflamatorios, hormonales y sociales. Las variables que se asocian con el envejecimiento exitoso con mayor evidencia son la edad, los trastornos de

\section{Introdução}

Na medida em que a expectativa de vida aumenta em diversos países, espera-se uma marcante expansão dos níveis de morbidade em termos populacionais. Portanto, o desafio para o futuro consiste em se promover o aumento da expectativa de vida saudável e livre de incapacidades.

Se considerarmos o fato de que a trajetória do envelhecimento é extremamente variável e que a deterioração não é inevitável, o termo "envelhecimento bem-sucedido" (EBS) alcançou um papel proeminente na literatura gerontológica e tornou-se um modelo cada vez mais popularizado a partir de 1987, quando Rowe e Kahn propuseram a distinção entre o envelhecimento "normal" e o "bem-sucedido", 1 e posteriormente ${ }^{2}$ definiram critérios objetivos para a sua classificação. Porém, críticas emergiram diante desta proposta e, sob diferentes perspectivas (social, biomédica ou psicológica), surgiram outras múltiplas formas de se mensurar e operacionalizar este construto, gerando pelo menos 29 diferentes definições na literatura. ${ }^{3}$ A tendência atual é que ele seja mensurado de forma multidimensional, englobando critérios objetivos acerca de questões biomédicas, aspectos psicológicos e sociais, e também critérios subjetivos relacionados à perspectiva dos idosos relacionada à qualidade de vida. ${ }^{4}$

Dentre os construtos existentes, o modelo proposto por Rowe e Kahn é o mais popularizado e utilizado na literatura científica, incluindo três principais componentes: ausência de doenças e incapacidades; manutenção da capacidade física e cognitiva; e engajamento ativo com a vida.

Embora não exista um consenso para a definição, segundo Thomas Glass, existem alguns pontos de concordância entre os autores..$^{5} \mathrm{O}$ primeiro é que o envelhecimento bem-sucedido se refere a algo entre a longevidade e a saúde, está relacionado à boa saúde na idade avançada e à capacidade de funcionar em múltiplos domínios (cognitivo, social e emocional). Além disso, tem relação com o que os idosos valorizam como articulación, la audición, las actividades de la vida diaria y el tabaquismo. Conclusiones: Para implementar estrategias efectivas de envejecimiento exitoso, es necesario desarrollar un mejor conocimiento sobre los fatores predictivos: tanto aquellos relacionados con la tasa de declinación como los factores protectores responsables del mantenimiento de la vitalidad y la resistencia al estrés.

Palabras clave: Envejecimiento exitoso; Envejecimiento saludable; Predictores.

qualidade de vida e implica em envelhecer melhor do que o "envelhecimento usual", descrito por Rowe e Kahn, ${ }^{1}$ no qual há um declínio funcional e da saúde. Também é consenso que muitas alterações cognitivas e funcionais fisiológicas relacionadas à idade podem estar relacionadas a fatores modificáveis do estilo de vida. ${ }^{6}$

\section{Fatores preditores do envelhecimento bem-sucedido}

Em termos individuais, estima-se que o processo do envelhecimento seja determinado em $25 \%$ pela genética e em $75 \%$ por fatores extrínsecos. ${ }^{7}$ Existem múltiplos fatores ambientais, comportamentais, psicológicos inflamatórios, endócrinos e sociais capazes de modificar a taxa de envelhecimento.

Apesar de estudos transversais identificarem uma série de variáveis associadas ao EBS, a avaliação da extensão a que estas variáveis correspondem de fato a preditores tem sido limitada pela escassez de estudos longitudinais. Embora haja evidência em estudos transversais de que ser casado, ter um trabalho remunerado ou voluntário, ter hábitos saudáveis, como beber moderadamente, manter um índice de massa corporal baixo, praticar atividade física, não fumar, ter um suporte social adequado e manter fortes crenças religiosas estejam associados ao EBS, é incerto se tais variáveis são de fato preditores ou apenas características associadas ao EBS em um momento pontual de análise. Como esperado, as análises transversais têm identificado um número bem maior de preditores associados ao EBS quando comparado às análises longitudinais. ${ }^{8}$

Dentre os estudos longitudinais recentemente publicados, destaca-se o de Hsu eJones, ${ }^{9}$ que encontrou como fatores associados a escolaridade, idades mais jovens, sexo masculino, ser morador da zona rural e ser casado. Já o estudo de Pruchno e Wilson-Genderson, ${ }^{8}$ mostrou que características identificáveis precocemente ao longo da vida (sexo, raça, educação, isolamento, nunca se casar), bem como fatores da vida 
adulta (manter-se casado, trabalhar), estilo de vida (tabagismo, etilismo, índice de massa corpórea e exercícios) e suporte social, distinguiram indivíduos que permaneceram bem-sucedidos ao longo de 4 anos de acompanhamento.

O estudo americano longitudinal MacArthur studies of successful ageing, conduzido em $1988,{ }^{10}$ é o estudo mais conhecido e publicado relacionado à dimensão biomédica do EBS. Um dos resultados mais importantes deste estudo consistiu na identificação de fatores potencialmente modificáveis (atividades físicas, suporte social e senso de autoeficácia) que pudessem influenciar os padrões de desempenho físico. Dentre outros estudos longitudinais, destacamos o Alameda County study, ${ }^{11}$ que encontrou associação com cor da pele, renda, níveis tensionais, artrite e lombalgia, tabagismo, massa corporal e etilismo. Nesta mesma coorte, um estudo posterior $^{12}$ evidenciou associação com os fatores escolaridade, raça, diabetes, DPOC, artrite, deficit auditivo, ausência de depressão, amizades e atividade física. Já o Study of Manitoba elderly, ${ }^{13}$ evidenciou associação com idade, ausência de câncer, boa autopercepção de saúde, aposentadoria por doença, ausência de diabetes e doença mental.

Em uma metanálise que incluiu 28 estudos $^{3}$ englobando diferentes definições para o EBS, as variáveis mais consistentes (de forte evidência) foram: idade jovem (próxima aos 60 anos), ausência de artrite, ausência de problemas auditivos, independência para as atividades de vida diária e não ser tabagista. Dentre as variáveis com evidência moderada destacaram-se os altos níveis de atividade física/exercício, melhores níveis de saúde autorrelatada, baixos níveis pressóricos, menor número de comorbidades, função cognitiva global e ausência de depressão. Apesar de outras publicações mostrarem a relação entre variáveis socioemográficas e mortalidade, incapacidade e doenças, nesta metanálise, tais variáveis (gênero, estado civil, etnia, recursos financeiros e escolaridade), apresentaram evidências limitadas nesta metanálise. ${ }^{3}$ Outras variáveis psicológicas, bem como marcadores biomédicos e atividades produtivas sociais, foram examinadas em número muito pequeno de estudos nesta metanálise, impossibilitando a análise do grau de evidência.

Contrariamente a esta metanálise, que avaliou estudos realizados apenas em países desenvolvidos encontrando evidências limitadas acerca da associação com variáveis socioeconômicas, um estudo brasileiro ${ }^{14}$ mostrou que variáveis socioeconômicas e psicossociais prevaleceram sobre as variáveis clínicas como predi- tores associados ao EBS, verificando-se a renda familiar e o número de confidentes como fatores protetores, e o número de crianças coabitando como fator de risco. Porém, este estudo analisou uma amostra de idosos saudáveis, o que pode ter minimizado a associação do EBS com variáveis clínicas.

\section{Fatores preditores e perspectiva de curso de vida}

Contrariamente a modelos que traçam uma avaliação estática do indivíduo na fase adulta tardia, a perspectiva de curso de vida é dinâmica e considera o desenvolvimento, a história e a importância dos relacionamentos ao longo do tempo. Além disso, reconhece que diversas experiências podem dar origem a distintas interpretações de sucesso, tanto em nível individual quanto cultural. ${ }^{15}$

A perspectiva de curso de vida reconhece influências para o desenvolvimento presentes antes mesmo do nascimento. Dentre essas influências iniciais estão a genética, além das condições e riscos ambientais (por exemplo, a pobreza) transmitidos através das gerações de uma família. Em uma análise multicêntrica, verficou-se que a desvantagem econômica na infância prediz negativamente o EBS na idade adulta. ${ }^{16}$ Além disso, Schafer e Ferraro, avaliaram o impacto das influências da infância (por exemplo, o abuso dos pais) e fatores comportamentais de adultos (por exemplo, tabagismo) sobre o estado "livre de doença" na velhice. Estes dois conjuntos de influências foram comparáveis em força de predição deste critério na idade avançada. ${ }^{16}$

Existem estudos mostrando modificação de risco para a saúde associado a mudanças comportamentais. Por exemplo, Pruchno e colaboradores, compararam tabagismo ativo e prévio para determinar se a cessação do tabagismo e o seu momento afetariam a classificação do EBS dentre adultos. A cessação do tabagismo aumentou as chances de uma classificação como bem-sucedido dentre ex-fumantes em relação aos fumantes atuais, mas apenas para os que cessaram o tabagismo antes dos 30 anos de idade. O impacto da exposição cumulativa foi evidente, reduzindo a probabilidade de EBS. ${ }^{8}$

Conforme discutido, as circunstâncias familiares, os relacionamentos e eventos representam poderosas influências para o desenvolvimento precoce, com potenciais consequências a longo prazo. A influência dos contatos sociais para o desenvolvimento e para o envelhecimento provavelmente se ampliam à medida em que os indivíduos entram na idade adulta e os contatos sociais se expandem. Christakis e seus colegas 


\section{Artigo de revisão}

documentaram o papel significativo das conexões sociais sobre comportamentos relacionados à saúde (por exemplo, comer, fumar e comportamentos sexuais de risco) e os desfechos de saúde. ${ }^{17}$ Eles verificaram que a influência das conexões sociais se estendem até três graus (ou seja, de amigos de amigos de amigos) e que algumas condições de saúde são influenciadas mais pelos amigos do que por relações mais estreitas, tais como cônjuges. No entanto, a qualidade das relações conjugais para o bem-estar de adultos está firmemente estabelecida. Tanto dentre os casais jovens quanto dentre os mais velhos os padrões de interações conjugais são conhecidos por influenciar o estado de saúde, sintomas físicos e condições crônicas. Cônjuges também afetam o engajamento social mútuo. ${ }^{18}$

As diferenças culturais influenciam na forma como os indivíduos vêem a sua saúde, o que eles consideram importante na vida e o significado de "sucesso". Em alguns estudos sobre envelhecimento saudável, foi verificado que os domínios pessoal, familiar e espiritual da vida foram mencionados mais em classificações leigas do que naquelas acadêmicas, e que os laços familiares e a estabilidade financeira desempenharam um papel único nas culturas orientais. ${ }^{20}$

As particularidades locais de diferentes países destacam elementos macrossociais que podem influenciar no processo de envelhecimento. Usando uma definição de EBS semelhante à de Rowe e Kahn, autores ${ }^{21}$ documentaram taxas dramaticamente mais elevadas de EBS em países do Norte da Europa (por exemplo, 21\% na Dinamarca) com relação ao Sul (3,1\% em Espanha) e a Europa Oriental (1,6\% na Polônia), refletindo diferenças nacionais de recursos e provisão de bem-estar. Assim, as políticas públicas que contribuem para a desigualdade de renda afetam as taxas de EBS no nível macro, independentemente das características e estilos de vida pessoais dos indivíduos. ${ }^{21}$

\section{Fatores genéticos}

A contribuição genética para a longevidade tem sido estimada utilizando-se tanto amostras de gêmeos quanto amostras populacionais. A hereditariedade da longevidade estimada em estudos com gêmeos varia entre $20 \%$ e $30 \%$, e em amostras populacionais é ligeiramente menor, variando entre $15 \%$ e $25 \%{ }^{22}$ Usando os dados do projeto GenomeEUtwin, que incluiu mais de 20.000 gêmeos nórdicos, Hjelmborg e colaboradores observaram que os efeitos genéticos sobre a expectativa de vida eram mínimos antes dos 60 anos de idade, mas foram significativos naqueles com mais de 60 anos, apresentando aumento constante e paralelo ao aumento da idade..$^{23}$

O envelhecimento saudável também possui influência hereditária. Reed e Dick ${ }^{24}$ definiram 'bem-estar' em gêmeos do sexo masculino, como atingir a idade de 70 anos livre de doenças cardíacas, cirurgia, acidente vascular cerebral, diabetes ou neoplasia da próstata; e mostrou que esta característica apresentava uma hereditariedade de 50\%.

\section{Conclusões}

Uma melhor compreensão do impacto de fatores extrínsecos sobre a senescência é fundamental para a implementação de estratégias de promoção do envelhecimento bem-sucedido, porém, é importante que se considere a influência destes determinantes ao longo de todo um curso de vida. Identificar intervenções eficazes para condições relacionadas à idade avançada torna-se difícil sem o emprego de uma visão do envelhecimento a longo prazo. Para as condições modificadas diretamente pela exposição precoce durante períodos críticos, ou através de exposição cumulativa desencadeada por eventos e condições iniciais, é irrealista pensar que mudanças de estilo de vida, de modo isolado, possam reverter o problema.

\section{Referências}

1. Rowe JW, Kahn RL. Human aging: usual and successful. Science. 1987 Jul 10;237:143-9.

2. Rowe JW, Kahn RL. Successful aging. Aging (Milano). 1998 Apr;10:142-4.

3. Depp CA, Jeste DV. Definitions and predictors of successful aging: a comprehensive review of larger quantitative studies. Am J Geriatr Psychiatry. 2006 Jan;14(1):6-20.

4. Bowling A, lliffe $S$."Which model of successful ageing should be used? Baseline findings from a British longitudinal survey of ageing." Age Ageing. 2006;35(6):607-614.

5. Glass, TA. Assessing the success of successful aging. Ann Intern Med. 2003 Sep 2;139(5):382-3.

6. Franklin NC, Tate CA. Lifestyle and Successful Aging: An Overview. Am J Lifestyle Med. 2009 January 1;3(1):6-11.

7. Fernandez-Ballesteros R, Robine JM, Walker A, et al. Active aging: a global goal. Curr Gerontol Geriatr Res. 2013;298012.

8. Pruchno RA, Wilson-Genderson MA. Longitudinal Examination of the Effects of Early Influences and Midlife Characteristics on Successful Aging. J Gerontol B Psychol Sci Soc Sci. 2014 May 13.

9. Hsu HC, Jones BL. Multiple trajectories of successful aging of older and younger cohorts.Gerontologist. 2012;52:843-856.

10. Berkman LF, Seeman TE, Albert TM, et al. High, usual and impaired functioning in community-dwelling older men and women: findings from the MacArthur Foundation Research Network on Successful Aging. J Clin Epidemiol. 1993 Oct;46(10):1129-40.

11. Guralnik JM, kaplan GA. Predictors of healthy aging: prospective evidence from the Alameda County study. Am J Public Health. 1989 Jun;79(6):703-8. 
Ana Cristina Canêdo e Roberto A. Lourenço. • Determinantes do envelhecimento bem-sucedido

12. Strawbridge WJ, wallagen ML. Successful aging and wellbeing: Self-rated compared with Rowe e Kahn. Gerontologist. 2002;42(6):727-883.

13. Roos NP, havens B. Predictors of successful aging: a twelveyear study of Manitoba elderly. Am J Public Health. 1991 Jan; 81(1):63-8.

14. Chaves $M L$ et al. Predictors of normal and successful aging among urban-dwelling elderly Brazilians. J Gerontol B Psychol Sci Soc Sci. 2009 Sep; 64(5):597-602.

15. Stowe JD, Cooney TM. Examining Rowe and Kahn's Concept of Successful Aging: Importance of Taking a Life Course Perspective. Gerontologist. 2014 Jun 6.

16. Brandt M, Deindl C, Hank K. Tracing the origins of successful aging: The role of childhood conditions and social inequality in explaining later life health. Soc Sci Med. 2012; 74:1418-1425.

17. Schafer MH, Ferraro KF. Childhood misfortune as a threat to successful aging: Avoiding disease. Gerontologist. 2012;52:11-120.

18. Christakis NA, Fowler JA. Connected: The surprising power of our social networks and how they shape our lives. NewYork: Little Brown and Company. 2009.

19. Bookwala J. The role of marital quality in physical health during the mature years. Journal of Aging and Health. 2005;17:85-104.

20. Hung L, Kempen GIJM, Devries NK. Crosscultural comparison between academic and lay views of healthy ageing: A literature review. Ageing Soc. 2010;30:1371-1391.

21. Brandt M, Deindl C, Hank K. Tracing the origins of successful aging: The role of childhood conditions and social inequality in explaining later life health. Soc Sci Med. 2012;74:1418-1425.

22. Murabito J, Yuan R, Lunetta K. The Search for Longevity and Healthy Aging Genes: Insights From Epidemiological Studies and Samples of Long-Lived Individuals. J Gerontol A Biol Sci Med Sci. 2012;67A(5):470-479.

23. Hjelmborg J, lachine I, Skytthe A. Genetic influence on human lifespan and longevity. Hum Genet. 2006;119(3):312-321.

24. Reed T, Dick D. Heritability and validity of healthy physical aging (wellness) in elderly male twins. Twin Res. 2003;6:227-234. 